\title{
Behavioral risk elicits selective activation of the executive system in adolescents: clinical implications
}

\author{
Richard H. Yaxley ${ }^{1}$, Elizabeth E. Van Voorhees ${ }^{1}$, Sara Bergman ${ }^{2}$, Stephen R. Hooper ${ }^{1,3}$, Scott A. Huettel ${ }^{2,4}$ \\ and Michael D. De Bellis ${ }^{1,4}$ *
}

\author{
' Healthy Childhood Brain Development Developmental Traumatology Research Program, Department of Psychiatry and Behavioral Sciences, Duke University, \\ Durham, NC, USA \\ ${ }^{2}$ Center for Cognitive Neuroscience, Duke University, Durham, NC, USA \\ ${ }^{3}$ Department of Psychiatry and Carolina Institute for Developmental Disabilities, University of North Carolina at Chapel Hill, Chapel Hill, NC, USA \\ ${ }^{4}$ Department of Psychology and Neuroscience, Duke University, Durham, NC, USA
}

\section{Edited by:}

Josephine Johns, University of North Carolina at Chapel Hill, USA

\section{Reviewed by:}

Marc N. Potenza, Yale University, USA

Duncan B. Clark, University of

Pittsburgh, USA

\section{*Correspondence:}

Michael D. De Bellis, Department of Psychiatry and Behavioral Sciences, Duke University Medical Center, Box 104360, Durham, NC 27710, USA. e-mail:debel002@mc.duke.edu
We investigated adolescent brain processing of decisions under conditions of varying risk, reward, and uncertainty. Adolescents $(n=31)$ preformed a Decision-Reward Uncertainty task that separates decision uncertainty into behavioral and reward risk, while they were scanned using functional magnetic resonance imaging. Behavioral risk trials involved uncertainty about which action to perform to earn a fixed monetary reward. In contrast, during reward risk the decision that might lead to a reward was known, but the likelihood of earning a reward was probabilistically determined. Behavioral risk trials evoked greater activation than the reward risk and no risk conditions in the anterior cingulate, medial frontal gyrus, bilateral frontal poles, bilateral inferior parietal lobe, precuneus, bilateral superior-middle frontal gyrus, inferior frontal gyrus, and insula. Our results were similar to those of young adults using the same task (Huettel, 2006) except that adolescents did not show significant activation in the posterior supramarginal gyrus during behavioral risk. During the behavioral risk condition regardless of reward outcome, overall mean frontal pole activity showed a positive correlation with age during the behavioral and reward risk conditions suggesting a developmental difference of this region of interest. Additionally, reward response to the Decision-Reward Uncertainty task in adolescents was similar to that seen in young adults (Huettel, 2006). Our data did not show a correlation between age and mean ventral striatum activity during the three conditions. While our results came from a healthy high functioning non-maltreated sample of adolescents, this method can be used to address types of risks and reward processing in children and adolescents with predisposing vulnerabilities and add to the paucity of imaging studies of risk and reward processing during adolescence.

Keywords: risk, behavioral risk, decision making, reward, adolescence, prefrontal brain regions, reward response, nucleus accumbens

\section{INTRODUCTION}

Adolescence represents a period of decision making that involves increased risk taking. Risk taking is defined as engaging in behaviors that may be high in subjective desirability (i.e., associated with high perceived reward) but which expose the individual to potential injury or loss (Geier and Luna, 2009). Examples of adolescent risk-taking include initiating use of alcohol and other addictive drugs (resulting in addiction) or engaging in unprotected sex (resulting in teenage pregnancies). The known increases in adolescent risk behaviors are observed across cultures (Spear, 2000) and associated with less mature prefrontal inhibitory control circuits (Ernst et al., 2006). Adolescent risk taking is a major public health concern whose negative results can lead to impaired maternal-infant interactions due to addictions and/or teen parenting. However, some risk taking may be normative, in that it allows for exploration of adult roles and for development of relevant coping skills (Siegel and Shaughnessy, 1995; Spear, 2000; Dahl, 2004; Kelley et al., 2004; Geier and Luna,
2009). Consequently, the neurobiological study of adolescent decision and reward processing using functional magnetic resonance imaging ( $\mathrm{fMRI}$ ) is timely.

Brain imaging studies have demonstrated that adolescents exhibit less activation in executive brain regions during decision making in gambling tasks than adults, which suggests an immaturity of these regions during adolescence (Eshel et al., 2007; Ernst and Mueller, 2008). In this investigation, we examined the neurodevelopmental maturity of adolescents using a novel task designed to challenge the dorsal lateral prefrontal executive control and ventral medial prefrontal reward circuits (Huettel, 2006). This Decision-Reward Uncertainty task separates decisions into behavioral risk and reward risk (Huettel, 2006). The DecisionReward Uncertainty Task represents an innovative approach to understanding decision making and reward. While most decisionmaking tasks used in addiction research combine decision making, response, and reward evaluation in time, the Decision-Reward Uncertainty Task was designed to examine decision making and 
reward circuits separately in one task (see Figure 1). Decisionmaking circuits involve a set of brain structures: prefrontal cortex; dorsolateral prefrontal cortex; parietal cortex; insular cortex; and anterior and posterior cingulate (Paulus et al., 2001; Huettel, 2006). Reward circuits involve a set of brain structures that receive dopaminergic input from the midbrain and include the ventral striatum (Vstr; which includes the nucleus accumbens), and ventromedial prefrontal cortex (Schott et al., 2008).

The Decision-Reward Uncertainty task is an advance because most previous research failed to differentiate decisions into risk types (i.e., reward risk versus behavioral risk) and reward response (Bolla et al., 2005; Huettel et al., 2005; Verdejo-Garcia et al., 2007) Thus, in most studies, decision making (also called response selection) was contingent in time upon reward and not separated from reward delivery (Xiangrui et al., 2010). Reward risk is defined as certainty about behavior but uncertainty about possible outcomes (i.e., reward presence). In other words, one knows what actions to take for a reward but the probability of reward is not certain. Reward risk activates reward circuits in the ventromedial prefrontal cortex, striatum, and other subcortical components of reward networks (Huettel, 2006). Behavioral risk is defined as uncertainty about which decisions and actions should be taken to earn a reward or achieve a desired goal. Under these conditions, one does not know what actions to take for a reward. The Decision-Reward Uncertainty Task examines three types of risk: reward risk, behavioral risk, and no risk. In reward risk trials, the action required to earn a reward is known, but the outcome of each trial is probabilistic. In behavioral risk trials, there is limited knowledge about which action to take (i.e., button to press), and the participant chooses between two possible button presses, one of which randomly determines a reward on that trial. The only difference between these conditions is whether a subject knows the correct action (reward risk) or not (behavioral risk). In other words, in reward risk, the decision and action to take are certain and in behavioral risk, the decision and action to take are uncertain. The Decision-Reward Uncertainty Task includes a no risk or certainty condition as a control, where the action required to earn a reward is known and reward is certain.

While undergoing the Decision-Reward Uncertainty Task during the behavioral risk condition, healthy young adults activated executive-control circuits including the prefrontal, parietal, and insular regions, within which no effect of reward risk was observed (Huettel, 2006), Reward delivery, in comparison to no reward, evoked increased activity in the ventromedial prefrontal cortex and the Vstr which includes the nucleus accumbens (Huettel, 2006). In healthy young adults undergoing this task, reward risk activated nucleus accumbens and ventromedial prefrontal cortex suggesting that distinct brain systems are recruited for the resolution of these different forms of risk (Huettel, 2006).

However, the Decision-Reward Uncertainty Task results were derived from samples of young-adult participants, and it is not clear whether they generalize to adolescence, when the prefrontal cortex is actively undergoing maturational changes. Indeed, the dorsolateral prefrontal cortex completes its pruning of gray matter only toward the end of adolescence (Gogtay et al., 2004). Consequently, conclusions about decision processes derived from adult

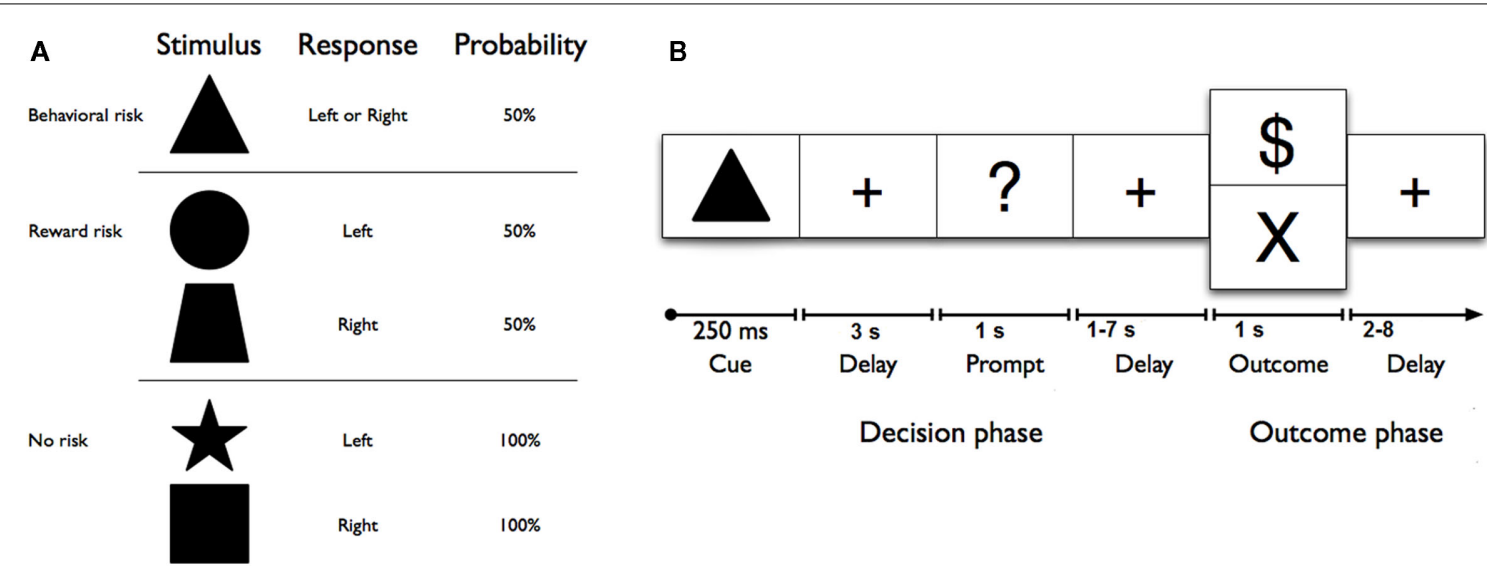

FIGURE 1 | (A) Geometric shape cues, button press response(s), and probabilities of reward for each risk condition. No risk cues (left button press on right hand for a star, or right button press on right hand for a square) signaled that the known behavioral response would be rewarded with $100 \%$ certainty. Reward risk cues (right button press for a trapezoid, or left button press for a circle) signaled that the known behavioral response would be rewarded with $50 \%$ probability. However, the behavioral risk cue (a triangle) signaled that the behavioral response was unknown; on each trial, either one of the two possible responses would be guaranteed a reward (" $\$$ or $\$ \$$ (not shown))" while the other would not. (B) Sequence of events used in each trial of the reward uncertainty task. A shape cue marked the start of each trial. After a fixed interval, a response prompt was presented. Participants were asked to press one of two buttons using their right hand as soon as the prompt appeared to make their choice. The outcome of each trial was determined by both (a) a correct right or left button response and (b) a probabilistically determined reward. Thus, each trial began with a shape cue for $250 \mathrm{~ms}$ in the center of the screen that indicated the trial type. After a 3-s delay, participants were prompted (i.e., "?") for $1 \mathrm{~s}$ to indicate their choice with a left or right button press with the second or third finger on the right hand. After a jittered delay (1, 3, 5, or $7 \mathrm{~s})$ where the fixation cross was presented, the trial outcome (reward: "\$" for no and reward risk, reward: "\$\$" (not shown here) for behavioral risk, or no reward: " $x$ ") was presented for $1 \mathrm{~s}$, and an updated tally of cumulative earnings was displayed in the lower portion of the screen. A fixation cross was displayed in the center of the screen during a jittered inter-trial interval $(2,4,6$, or $8 \mathrm{~s})$. Participants completed 150 trials on average, split evenly among six 6-min runs. Optimal performance could yield up to an additional \$25 (e.g., \$0.15 per correct response; for one dollar sign; $\$ 0.30$ for two dollar signs). 
samples may not generalize well to adolescents. Given the differences in behavior and levels of brain maturation in adolescents and adults, an important question for current research is whether these differences are evident in both behavioral and reward risk, and their brain circuitry regions of interest. To date, previous studies have not investigated how different types of risk are represented in adolescent executive and reward networks. This is a potentially important distinction, because it may have social and policy implications. We hypothesize that in adolescents, behavioral risk will activate executive-control circuits their associated functional regions of interests while reward risk will activate reward circuits and their associated functional regions of interest as suggested in the Huettel (2006) study. However, in this study, we wished to examine in adolescents the neural correlates of decision making with respect to reward and behavioral risks. Furthermore, we predict an association with executive control and reward circuits regions of interest and age.

\section{MATERIALS AND METHODS PARTICIPANTS}

Thirty-one healthy adolescents (mean age and SD: $15.5 \pm 1.5$ years; age range: $12.3-17.7$ years; 21 females, 10 males) participated in a detailed clinical research assessment, and then engaged in the Decision-Reward Uncertainty Task while undergoing fMRI on another day. There were no gender or age differences (mean age females $15.5 \pm 1.6$, mean age males $15.6 \pm 1.2$ years: $F=0.01$, $\mathrm{df}=21, p=0.94)$ in the control group. Healthy adolescent participants were recruited from the community by IRB approved advertisements. Adolescents provided written assent and legal guardians provided written informed consent before participation. Male and females did not differ in handedness, IQ, or socioeconomic status. All participants came from a range of socioeconomic environments (middle to upper socioeconomic strata).

The clinical assessment portion of the study was undertaken at the Healthy Childhood Brain Development Developmental Traumatology Research Program and included interviews of both adolescents and their legal guardians using the Schedule for Affective Disorders and Schizophrenia for School Aged Children Present and Lifetime Version (KSADS-PL), which includes a comprehensive post-traumatic stress disorder interview (Kaufman et al., 1997). This semi-structured interview was administered to caregivers and adolescents. We also used archival records as additional sources of information. The KSADS-PL was modified to include additional information about: (1) life events, including traumatic events from the Child and Adolescent Psychiatric Assessment (Angold et al., 1995); and (2) disorders not present in the KSADSPL. Modifications also included: (3) an added structured scale to quantify symptom frequency with a minimum score of $0=$ no history of a symptom and maximum score of $10=$ symptoms present several times a day; and (4) algorithms to determine Axis I psychiatric disorders based on DSM-IV criteria. Disorders were assigned a severity score of mild, moderate, or severe. This modified version is available upon request. Interviewers were individually trained to obtain over $90 \%$ agreement for the presence of any lifetime major Axis I disorder with a board certified child and adolescent psychiatrist and experienced child trauma interviewer (MDDB). Discrepancies were resolved by reviewing archival information (e.g., school records, birth, and pediatric medical records) or by re-interviewing the child or caregiver. If diagnostic disagreements were not resolved with this method, consensus diagnoses were reached among a child psychiatrist (MDDB) and child psychologist (SRH). Subjects also underwent extensive neuropsychological testing to verify that they were age-typical. This included a twosubtest short-form of the Wechsler Intelligence Scale for ChildrenIII (WISC-III; Wechsler, 1991) comprised of Vocabulary and Block Design, to generate an IQ score. Mean IQ was $113.1 \pm 11.0$ (IQ range 90-132). Adolescents also received saliva and urine toxicology screens to confirm the absence of alcohol, tobacco, or other drug use on the day of interview and imaging data collection. Participants with an Axis I diagnosis, who were not age-typical on neuropsychological testing or had a positive alcohol or drug screen, were excluded.

Exclusion criteria for subjects were: (1) current or lifetime history of DSM-IV Axis I psychiatric disorders including alcohol and substance use disorders, (2) significant medical, neurological, or psychiatric disorder, (3) history of head injury or loss of consciousness, (4) pregnancy, (5) history of prenatal or birth confounds that could have influenced brain maturation such as significant prenatal exposure to substances, severe birth complications, or birth weight under $5 \mathrm{lb}$ or severe postnatal compromise with neonatal intensive care unit (NICU) stay; (6) morbid obesity or growth failure, (7) full scale IQ lower than 90, (8) history of trauma or child maltreatment, or (9) contraindications to safe participation in MRI research. The Institutional Review Board of the Duke University Medical Center approved this study.

\section{EXPERIMENTAL DESIGN}

We used an experimental paradigm, the Decision-Reward Uncertainty task, that we have used previously to examine neural correlates of risky decision making in young-adult participants (Huettel, 2006). Critically, the task was designed to temporally isolate three phases of decision making: (1) choice selection, (2) action execution, and (3) outcome or reward evaluation (Ernst and Paulus, 2005; Rangel et al., 2008). Our analyses focus on the initial choice selection and outcome evaluation phases of decision making.

In this task, we manipulated two types of risk: Reward risk and behavioral risk. In reward risk trials, the action required to earn a reward was known to the participant, but the outcome of each trial was probabilistic: if the correct button was pressed, there was a $50 \%$ probability of a reward. In behavioral risk trials, the participant chose between two possible button presses, one of which (randomly determined) guaranteed a reward on that trial. Note that the behavioral risk and reward risk conditions were matched on probability and expected value, in that each contained a $50 \%$ chance of receiving a constant-size reward. The only difference between these conditions was in whether the participant knew the correct action (reward risk) or not (behavioral risk). We also included a no risk condition as a control. In the no risk condition, the action required to earn a reward was known and the likelihood of earning a reward was certain.

Each condition was represented by a visual cue (square, star, circle, trapezoid, or triangle) and mapped directly to a response [left (second digit) or right (third digit) button press with right hand; 
see Figure 1A]. No risk cues (left button press on right hand for a star, or right button press on right hand for a square) signaled that the known behavioral response would be rewarded with 100\% certainty. Reward risk cues (right button press for a trapezoid, or left button press for a circle) signaled that the known behavioral response would be rewarded with $50 \%$ probability. However, the behavioral risk cue (a triangle) signaled that the behavioral response was unknown; on each trial, either one of the two possible responses would be guaranteed a reward while the other would not. Each trial (Figure 1B) began with a shape cue for $250 \mathrm{~ms}$ in the center of the screen that indicated the trial type. After a 3-s delay, participants were prompted (i.e., "?") for $1 \mathrm{~s}$ to indicate their choice with a left or right button press with the second or third finger on their right hand. After a jittered delay $(1,3,5$, or $7 \mathrm{~s})$ where the fixation cross was presented, the trial outcome (reward: “ $\$$ ” for no risk, and “ $\$ \$$ ” for behavioral risk or reward risk, or no reward: " $x$ ”) was presented for $1 \mathrm{~s}$, and an updated tally of cumulative earnings was displayed in the lower portion of the screen. A fixation cross was displayed in the center of the screen during a jittered inter-trial interval $(2,4,6$, or $8 \mathrm{~s})$. Participants completed 150 trials on average, split evenly among six 6-min runs. As a developmental adaptation for the younger adolescents, the duration of each run was reduced to six 6-min runs for the adolescent group from the 10 min used in the young-adult group (Huettel, 2006). This adaptation greatly improved adolescent cooperation with the task. Optimal performance could yield up to an additional \$25 (e.g., \$0.15 per correct response; for one dollar sign; $\$ 0.30$ for two dollar signs) above the regular compensation for participation. Participants were trained on the task's cue-response contingencies in a prior behavioral testing session before scanning. To minimize practice or learning effects, all subjects practice the task until they showed that they had mastered the rules of the Decision-Reward Uncertainty task.

The experiment was programmed in MATLAB (MathWorks ${ }^{1}$ ) using the Psychophysics Toolbox (Brainard, 1997). Stimuli were displayed on goggles at a video resolution of $800 \times 600$ pixels and an apparent field of view of approximately $20^{\circ}$. Responses were collected on a four-button box, where only the first two buttons were used.

\section{IMAGE ACQUISITION}

The fMRI data for our adolescent participants were acquired using a 3.0-T General Electric (Waukesha, WI, USA) scanner. Wholebrain images sensitive to blood-oxygenation-level-dependent (BOLD) contrast were acquired using a high-throughput $\mathrm{T}_{2}^{*}$-weighted spiral-in pulse sequence $(\mathrm{TR}=2 \mathrm{~s}, \mathrm{TE}=28 \mathrm{~ms}$, flip angle $=90^{\circ}$, 34 slices, voxel size: $3.75 \mathrm{~mm} \times 3.75 \mathrm{~mm} \times 3.8 \mathrm{~mm}$ ). Data were acquired in a series of six sessions, each comprising 180 volumes. We additionally acquired whole-brain high-resolution images using a $\mathrm{T}_{1}$-weighted $3 \mathrm{D}$ spoiled gradient-recalled sequence to aid in normalization and registration of the functional images.

\section{fMRI DATA ANALYSIS}

Functional images were analyzed using fMRI Expert Analysis Tool (version 5.98, Analysis Group, FMRIB, Oxford, UK). These images

\footnotetext{
${ }^{1}$ http://www.mathworks.com
}

were corrected for slice acquisition time (interleaved ascending), corrected for motion with MCFLIRT, normalized into the standard Montreal Neurological Institute stereotaxic space (MNI, Montreal, QC, Canada), and subjected to a high-pass filter (pass frequency $>1 / 100 \mathrm{~Hz}$ ). FSL's Brain Extraction Tool (BET) was used to exclude non-brain voxels from our analyses. Four volumes from the start of each session were discarded to allow image intensity to stabilize. First-level (i.e., within-run) regression analyses included three regressors time-locked to the onset of the decision phase, defined as first second from the onset of the stimulus, of each trial type (behavioral risk, reward risk, and no risk), one nuisance regressor for all responses, and one nuisance regressor for missed responses. Second-level analyses collapsed across runs, within each subject, using a fixed-effects model. Across-subjects comparisons used a random-effects model that included an additional regressor for between-group comparisons. All reported results, including figures and tables, show activation that survived a wholebrain cluster family wise error (FWE) correction with a voxelwise $z$-statistic threshold of 2.3 ( $p \leq 0.01)$.

To examine the relationship between maturation and brain region of interests (ROI), we used mean ROI BOLD activity of brain regions which showed significant differences in our third level analyses and correlated these with age using parametric statistics (Pearson's correlations) and jmp 9.0.2 2010 SAS Institute Inc). Before Pearson's correlations were applied, data was tested for fit to the normal distribution using the Goodness of Fit Test (i.e., Shapiro-Wilk $W$ Test) in jmp.

\section{RESULTS \\ BEHAVIORAL ANALYSIS}

Only correct responses performed within a 1-s window after the response prompt (e.g., "?") was displayed were included in the analyses. Mean response times were analyzed by condition: no risk $($ Mean $=0.448, \mathrm{SD}=0.082 \mathrm{~s})$, reward risk $($ Mean $=0.453$, $\mathrm{SD}=0.082 \mathrm{~s})$, and behavioral risk (Mean $=0.451, \mathrm{SD}=0.091 \mathrm{~s})$. Response times were submitted to a repeated measures analysis of variance and showed no main effect of condition, $F(2,29)=0.373$, $p=0.692$.

\section{REGIONS ACTIVATED BY BEHAVIORAL RISK IN ADOLESCENTS}

To identify the brain regions that support decision making under behavioral risk, we contrasted activation associated with decisions in the behavioral risk (i.e., choice selection) condition with the mean activation associated with decisions in the no risk and reward risk conditions. In our adolescent sample this contrast elicited significant activations in brain regions typically implicated in risky decision making: anterior cingulate, medial frontal gyrus, bilateral frontal poles and inferior parietal lobe, precuneus, bilateral superior-middle frontal gyrus, inferior frontal gyrus (IFG), and insula (Figure 2; Table 1 reports the peak voxels present using the $z$-statistic threshold of 2.3). Within these significant cluster of regions also included the dorsal lateral prefrontal cortex, anterior insula, and lateral parietal regions. This pattern of activation replicates the key results from the adult sample described by Huettel

\footnotetext{
${ }^{2}$ www.jmp.com
} 


\section{Adolescents: $\mathrm{BR}>\mathrm{RR}+\mathrm{NR}$}

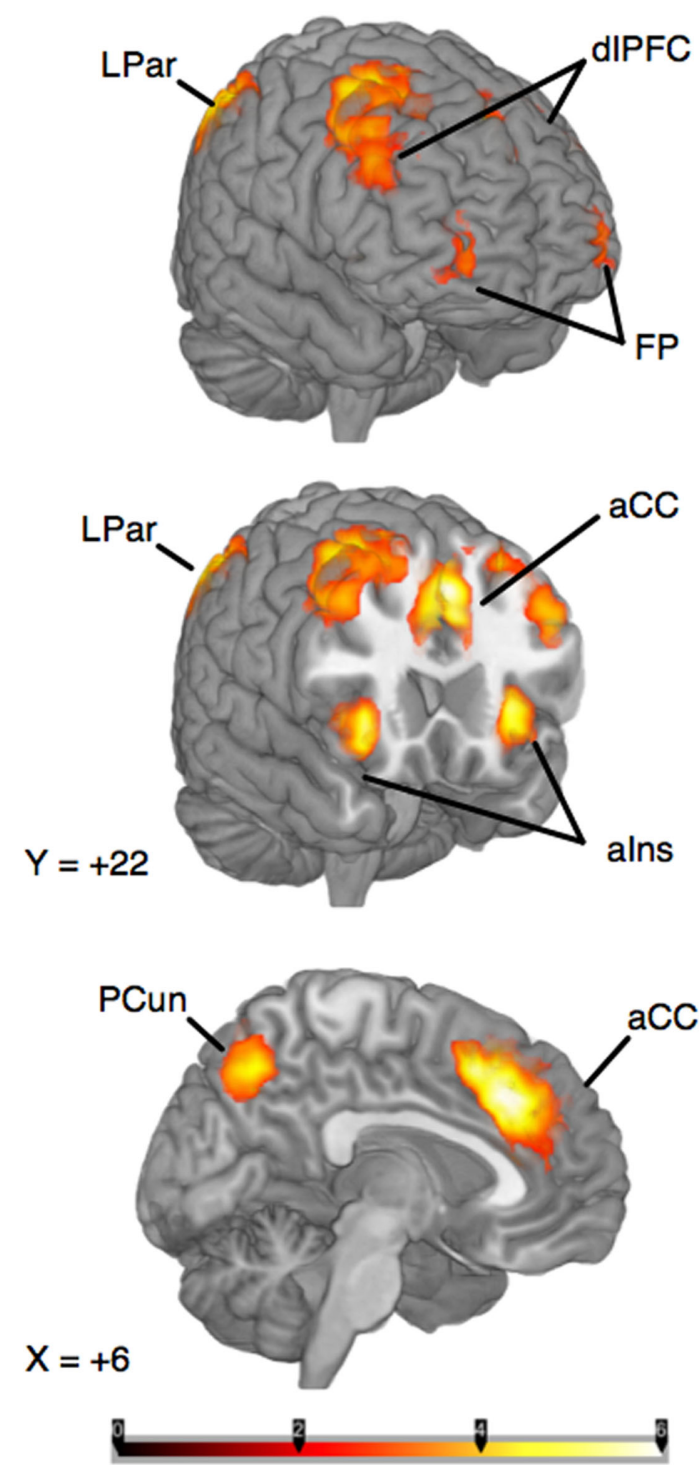

L

FIGURE 2 | Whole-brain analysis of decisions involving behavioral risk (BR) greater than reward risk (RR) and no risk (NR) conditions in adolescents. Behavioral risk elicited patterned activation in the insula, frontal poles (FP) and middle frontal gyrus, anterior cingulate (aCC), superior frontal gyrus, medial prefrontal cortex, precuneus (pCun), and Inferior Parietal Lobule. Within these significant cluster of regions also included the dorsolateral prefrontal cortex (dIPFC), anterior insula (alns), and lateral parietal regions (LPar).

(2006), indicating that adolescents activated the same decisionmaking network as adults during decisions involving behavioral risk. Mean percent signal change (SE) associated with the no risk (NR), reward risk (RR), and behavioral risk (BR) conditions are shown in Figure 3. Signal was extracted from two regions of interest: (Figure 3A) anterior cingulate and (Figure 3B) frontal pole.

\section{REGIONS ACTIVATED BY OUTCOME EVALUATION}

To distinguish the brain regions that responded to rewarding outcomes from those activated during decisions involving risk, we contrasted trials in both the reward risk and behavioral risk conditions that led to a rewarding outcome with those trials that led to no reward. When using $z$-statistic threshold $=2.3$, this contrast elicited large clusters of significant activations in three brain regions; (1) the IFG, middle frontal gyrus, and its sublobar areas; (2) the cingulate gyrus; and (3) the middle occipital gyrus. These include subclusters typically implicated in decision and reward processing: Vstr, which includes the nucleus accumbens and caudate, and putamen, and additionally activated the global pallidus and IFG, middle frontal gyrus, posterior cingulate, and large regions in the visual cortex. Because the peak activations in these regions were so large, we manually identified the subcluster in the Vstr and global pallidus. This is shown in Figure 4A and Table 2.

To examine the evoked activation in response to reward, we extracted the mean percent signal change in both rewarded and unrewarded conditions using an anatomically defined ROI in the Vstr which includes the nucleus accumbens (which was superimposed in green in Figure 4A). The nucleus accumbens was based on the standard ROI for the nucleus accumbens subcortical region as defined by the Harvard-Oxford atlas within FSL and is shown in green. Mean percent signal change in response to rewarded and unrewarded outcomes were calculated for each level of risk (Figure 4B).

\section{CORRELATIONS OF REGIONS OF INTEREST WITH AGE}

During the behavioral risk condition regardless of reward outcome, overall mean frontal pole BOLD activity showed a positive Pearson's correlation with age $(F=11.4, \mathrm{df}=29, p=0.002)$. See Figure 5A. Reward risk similarly showed a positive Pearson's correlation with age $(F=4.7, \mathrm{df}=29, p<0.04)$. See Figure 5B. These correlations suggest developmental differences during different types of decision making in the frontal pole with increasing age. We did not see significant correlations between age and mean anterior cingulate or mean Vstr BOLD activity with age.

\section{DISCUSSION}

We investigated functional brain activity in high functioning healthy adolescents while they performed the experimental Decision-Reward Uncertainty task (Huettel, 2006). We had two primary goals: to evaluate whether adolescents recruited the same decision-making network as young adults, and to examine whether the form of risk modulated these networks. Our goal was also to examine reward circuits and their regions of interest using the same simple task. Furthermore, we wanted to examine the association with executive control and reward circuits regions of interest with maturation measures (e.g., age). Our analyses focused on the decision making or choice selection and outcome evaluation phases of decisions that involved behavioral risk (i.e., decision making under uncertainty). Decision making during the task elicited activation in executive-control regions typically implicated in studies of adult decision making: frontal poles, anterior cingulate, superior, middle and medial prefrontal gyrus, precuneus, inferior parietal cortex, and insula (Huettel, 2006). Behavioral risk 
Table 1 | Cluster and subcluster activations for the decision phase: behavioral risk $>$ (no risk + reward risk).

\begin{tabular}{|c|c|c|c|c|c|c|}
\hline \multirow[t]{2}{*}{ Brain regions } & \multirow[t]{2}{*}{ Hemisphere } & \multirow[t]{2}{*}{ Voxel size $\left(\mathrm{mm}^{3}\right)$} & \multirow[t]{2}{*}{$z$} & \multicolumn{3}{|c|}{ MNI } \\
\hline & & & & $x$ & $y$ & $z$ \\
\hline Cingulate gyrus & $\mathrm{L}$ & & 6.23 & -4 & 18 & 44 \\
\hline Middle frontal gyrus & $\mathrm{R}$ & & 5.14 & 30 & 6 & 60 \\
\hline Middle frontal gyrus, frontal poles & $\mathrm{L}$ & & 5.09 & -42 & 28 & 32 \\
\hline Middle frontal gyrus & $\mathrm{R}$ & & 4.86 & 30 & 10 & 58 \\
\hline Inferior parietal lobe & $\mathrm{L}$ & 2020 & 5.41 & -44 & -64 & 44 \\
\hline Inferior parietal lobule & $\mathrm{L}$ & & 5.12 & -46 & -42 & 50 \\
\hline Inferior parietal lobule & $\mathrm{L}$ & & 5.07 & -38 & -58 & 46 \\
\hline Inferior parietal lobule & $\mathrm{L}$ & & 5.02 & -46 & -44 & 54 \\
\hline Inferior parietal lobule & $\mathrm{L}$ & & 5.01 & -38 & -60 & 54 \\
\hline Inferior parietal lobule & $\mathrm{R}$ & & 4.82 & 44 & -54 & 48 \\
\hline Inferior parietal lobule & $\mathrm{R}$ & & 4.7 & 44 & -60 & 44 \\
\hline Inferior parietal lobule & $\mathrm{R}$ & & 4.68 & 42 & -48 & 46 \\
\hline Inferior parietal lobule & $\mathrm{R}$ & & 3.79 & 42 & -38 & 44 \\
\hline Precuneus & $\mathrm{L}$ & 1648 & 4.78 & -10 & -68 & 38 \\
\hline Precuneus & $\mathrm{R}$ & & 4.75 & 2 & -64 & 48 \\
\hline Precuneus & $\mathrm{L}$ & & 4.7 & -2 & -62 & 48 \\
\hline Precuneus & $\mathrm{L}$ & & 4.57 & -8 & -64 & 46 \\
\hline Precuneus & $\mathrm{R}$ & & 3.47 & 14 & -64 & 58 \\
\hline Superior to middle frontal gyrus & $\mathrm{L}$ & 624 & 3.67 & -38 & 68 & -4 \\
\hline Middle frontal gyrus & $\mathrm{L}$ & & 3.59 & -32 & 64 & 6 \\
\hline Superior to middle frontal gyrus & $\mathrm{R}$ & 486 & 3.44 & 30 & 62 & -6 \\
\hline Middle frontal gyrus & $\mathrm{R}$ & & 3.37 & 34 & 64 & 10 \\
\hline Superior frontal gyrus & $\mathrm{R}$ & & 3.27 & 30 & 60 & -2 \\
\hline Superior frontal gyrus & $\mathrm{R}$ & & 3.25 & 32 & 64 & 2 \\
\hline Superior frontal gyrus & $\mathrm{R}$ & & 3.15 & 28 & 58 & 4 \\
\hline Middle frontal gyrus & $\mathrm{R}$ & & 2.9 & 38 & 58 & -10 \\
\hline
\end{tabular}

Shown for each cluster of significant activation $(Z>2.3)$ are the coordinates $\left(\mathrm{mm}^{3}\right.$ within standard Montreal Neurological Institute stereotaxic space (MNI) space) of the peak voxel within that cluster.

$L$, left, $R$, right.

trials, however, evoked greater activation than the other conditions in the anterior cingulate, dorsal lateral prefrontal cortex, frontal gyrus, frontal poles, inferior parietal lobe, precuneus, and anterior insula. Our results were similar to those of young adults using the same task (Huettel, 2006) except that adolescents did not show significant activation in the posterior supramarginal gyrus, a brain area involved in vocabulary and declarative memory (Lee et al., 2007), during behavioral risk. Our task does involve working memory (Huettel, 2006), a process that matures during adolescence. These findings show that choice selection during decisions involving behavioral risk elicits a network of brain regions including those that are involved in conflict monitoring (anterior cingulate; Kerns et al., 2004), visual attention (occipitoparietal cortex; Konrad et al., 2005), working memory and decision making (dorsolateral prefrontal cortex; Kwon et al., 2002; Huettel et al., 2005; Konrad et al., 2005), and interpreting the emotional significance and the intensity of stimuli (insula; for review see Ernst and Paulus, 2005). 
A

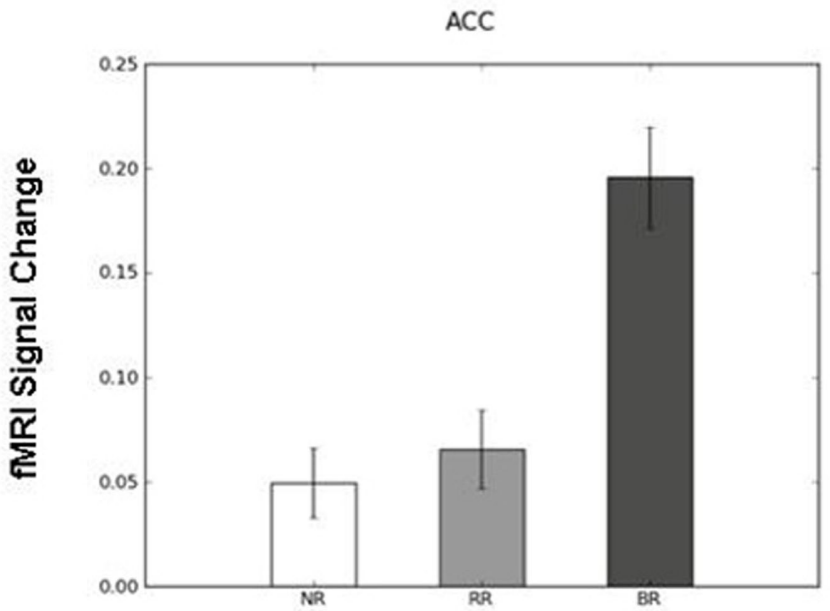

B

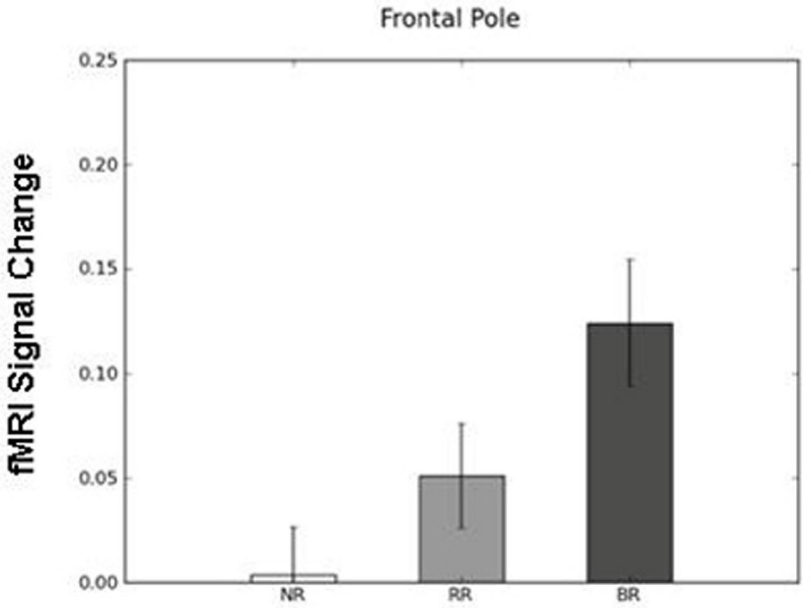

FIGURE 3 | Mean percent signal change (SE) associated with the no risk (NR), reward risk (RR), and behavioral risk (BR) conditions. Signal was extracted from two regions of interest: $(\mathbf{A})$ anterior cingulate and (B) frontal pole.

\section{A Rewarded outcomes for RR + BR}

IFG

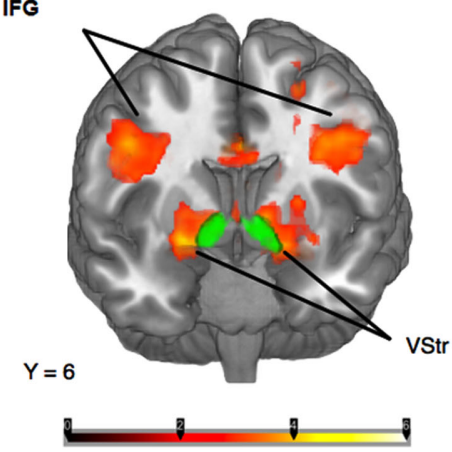

FIGURE 4 | (A) Whole-brain analysis of the outcome phase of the task for rewarded greater than unrewarded trials in the reward risk (RR) and behavioral risk (BR) conditions. Rewarded trials elicited greater activation in regions typically implicated in reward processing, such as the ventral striatum (Vstr), which includes the nucleus accumbens and caudate, and putamen, and additionally activated the global pallidus and inferior frontal gyrus (IFG). The

\section{B Signal change for RR + BR in Ventral Striatum}

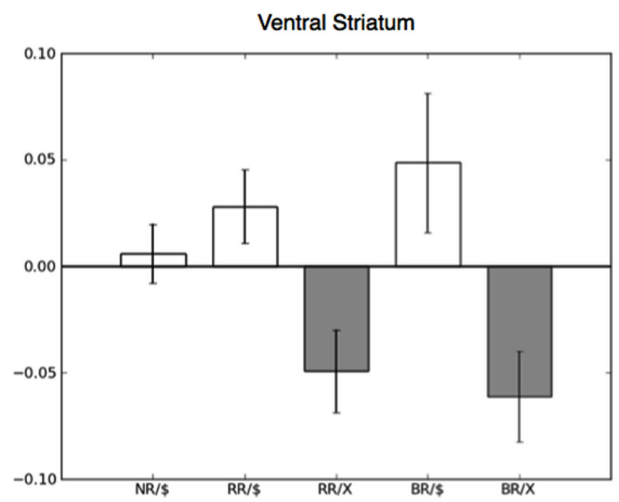

nucleus accumbens was based on the standard ROI for the nucleus accumbens subcortical region as defined by the Harvard-Oxford atlas within FSL and is shown in green. (B) Mean percent signal change extracted from an anatomically defined $\mathrm{ROI}$ in the Vstr. Mean percent signal change (SE) is plotted for rewarded no risk (NR), reward risk (RR), and behavioral risk (BR) outcomes, as well as unrewarded reward risk and behavioral risk outcomes.
In our results, reward versus no reward elicited significant activations in brain regions typically implicated in decision and reward processing (i.e., Vstr, inferior frontal, gyrus, anterior to middle cingulate, posterior cingulate, and visual cortex). Similar research findings were seen in studies of reward processing in primates (Apicella et al., 1991; Schultz et al., 2000; Roesch and Olson, 2004) and adults (O'Doherty et al., 2001; Delgado et al., 2003; Elliott et al., 2003). Reward response to the DecisionReward Uncertainty task was similar to that seen in young adults using this task (Huettel, 2006). There are few imaging studies of reward processing during adolescence. Those investigations also implicate neurocircuitry similar to those reported during reward response to the Decision-Reward Uncertainty task here, in that differences in BOLD activity were seen in visual cortex, Vstr, and anterior to middle cingulate during reward processing in adolescents (Bjork et al., 2004, 2007; May et al., 2004; Ernst and Paulus, 2005; Galvan et al., 2006; Eshel et al., 2007).

However, the reward response processing during adolescence is controversial, where some investigators believe the adolescent reward processing circuitry is hyporesponsive to rewards compared to those of adults (Spear, 2000), while others believe adolescents' reward circuits are hyperresponsive to rewards compared to adults (Chambers et al., 2003; Ernst et al., 2006). In a hypoactive 
Table 2 | Peak activations for the outcome phase: peak activations for reward $>$ no reward.

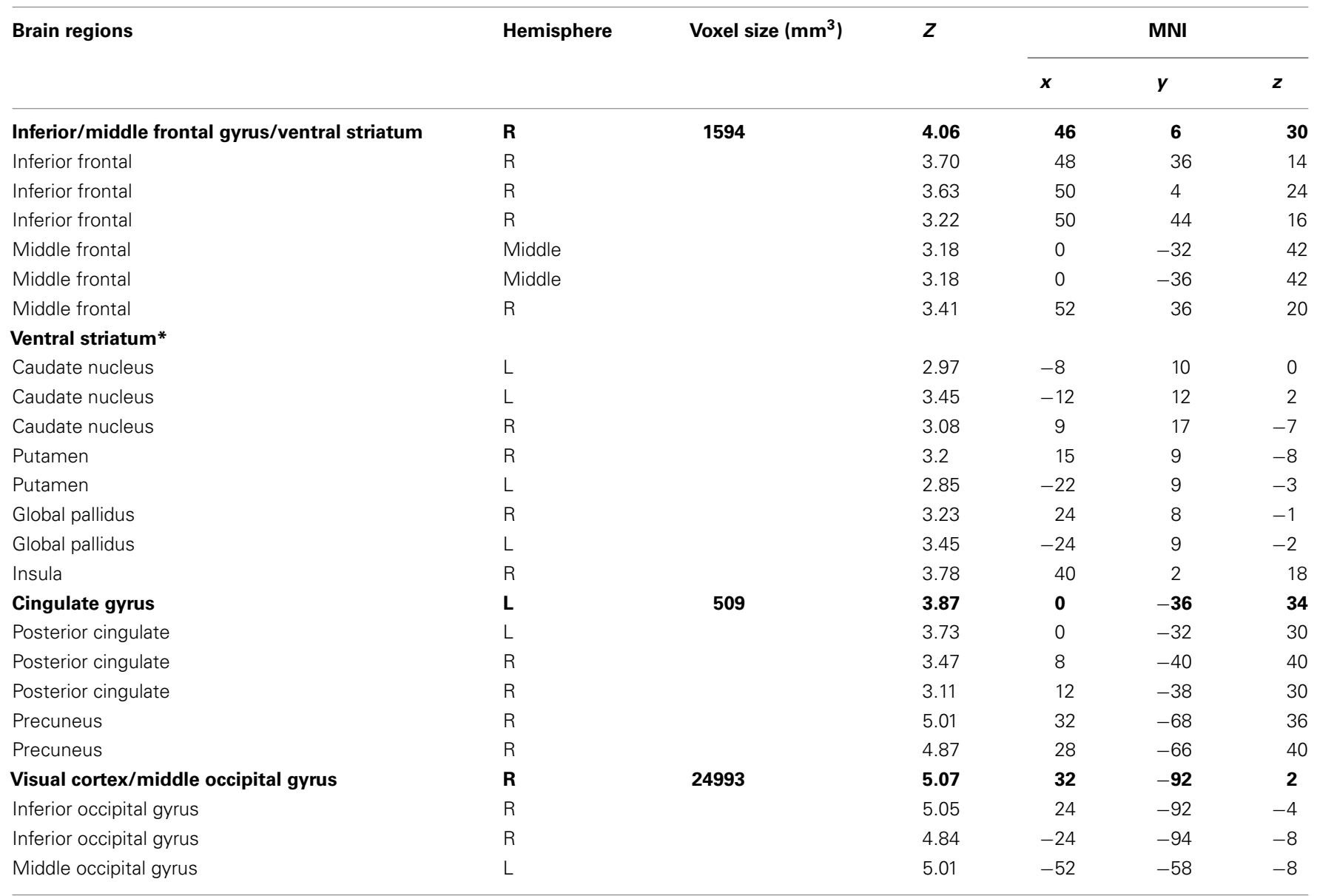

Shown is each cluster and subclusters of significant peak activations $(Z>2.3)$. The coordinates $\left(\mathrm{mm}^{3}\right)$ are within standard Montreal Neurological Institute stereotaxic space (MNI) of the peak voxel within that cluster.

$L$, left, $R$, right.

*The peak regions of activation in the subclusters of the right and left ventral striatum and global pallidus were manually identified.

A

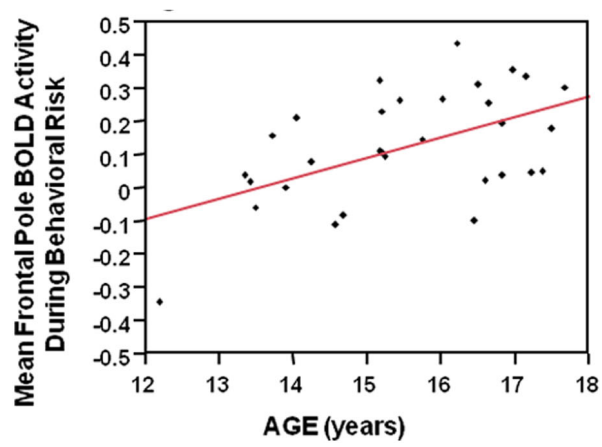

FIGURE 5 | (A) During the behavioral risk condition, regardless of reward outcome, overall mean frontal pole BOLD activity showed a positive Pearson's correlation with age ( $F=11.4, \mathrm{df}=29, p=0.002)$. (B) During the Reward risk condition, regardless of reward outcome overall mean frontal
B

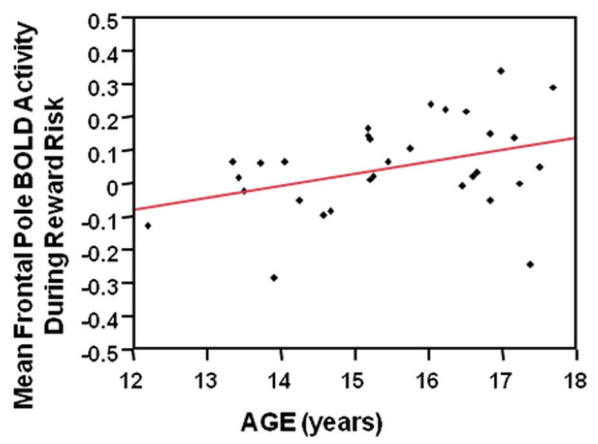

pole BOLD activity showed a positive Pearson's correlation with age $(F=4.7, \mathrm{df}=29, p<0.04)$. These correlations suggest developmental differences, during two different types of decision making, in the frontal pole with increasing age. 
reward processing system, brain areas that process rewards are not recruited as strongly as they are in adults. Our data did not provide support for this theory in that during the no risk condition, mean Vstr activity showed no positive correlation with age in carefully screened and comprehensively assessed healthy adolescents.

Decision making and reward processing in clinical populations is vastly understudied. Although speculative, a developmentally decreased sensitivity to executive function such as decision making under conditions of uncertainty may, in vulnerable adolescent populations, contribute to differences in reinforcement-related learning that lead to adolescent onset alcohol and substance use disorders (for review see Spear, 2000). For example, adult studies have also shown reduced activation in control and reward processes in abstinent cannabis users (Martin-Santos et al., 2010). Additionally, childhood adverse life events are associated with basal ganglia hyporesponse during fMRI evaluation of reward (Dillon et al., 2009; Mehta et al., 2010) which may further contribute to the known risk for adolescent onset alcohol and substance use disorders seen in victims of maltreatment (Anda et al., 1999; Kilpatrick et al., 2000). Preclinical studies suggest stress in young animals lowers dopamine D2 receptors in reward regions (Papp et al., 1994; Morgan et al., 2002), making animals and humans more vulnerable to addiction (De Bellis, 2001). One pediatric study, however, showed that while undergoing The Wheel of Fortune task, maltreated children with depression selected safe over risky options more frequently in the high-risk condition than did control children (i.e., they avoided selecting a large reward paired with a low chance of winning compared with maltreated children without depression and non-maltreated controls; Guyer et al., 2006). These limited data suggest that the effects of early familial adverse experiences or familial vulnerability on development of decision making and reward evaluation require further study as immaturity in executive decision making or reward systems may lead to substance use disorders and thus negatively influence the quality of care an addicted parent is able to provide.

Adolescence is a period during which the constituents of cognition develop to enable adaptive goal-directed behavior (for review see Ernst and Mueller, 2008). However, the emotional intensity also associated with adolescence influences the response to rewards that may contribute to increased risk-taking behaviors. Another model of adolescent reward processing suggests that adolescents demonstrate a heightened sensitivity to rewards and over active reward system. This "triadic model" proposes three behavioral control systems (approach, avoidance, and supervisory control systems) that differ between adolescents and adults (for review see Ernst et al., 2006). In other words, normative maturational increases in dopamine neurotransmitter activity in the frontostriatal "motivational" system coupled with relatively lower levels of inhibitory (e.g., serotoninergic) mechanisms in prefrontal systems contribute to increased reward sensitivity in adolescents (Chambers et al., 2003) and the known increases in normative adolescent risk behaviors (Dahl, 2004; Kelley et al., 2004). Thus the hyper-responsivity reward processing theory suggests that an overactive Vstr is unchecked by immature prefrontal inhibitory mechanisms. In our study, the behavioral and reward risk conditions regardless of reward outcome, showed a positive correlation with age and overall mean frontal pole activity. During the reward risk condition, mean frontal pole activity also showed a positive correlations with age. Our data suggest that the prefrontal system is immature at younger ages regardless of type of risk (behavioral or reward risk) and provide no direct support for the hyper-responsivity reward processing theory. However, immaturity of prefrontal executive supervisory control systems alone may account for dysregulation of reward processing during adolescence. Its activity matures from childhood to adulthood in parallel with increased capacity for adults to make healthy mature decisions (Eshel et al., 2007).

The relationship between reduced frontal pole activation and younger age during both the behavioral and reward risk conditions may mean that less reinforced risky rewards signal the availability of reinforcement in adolescents. As greater reward was associated with greater risk in this task, a developmentally immature and less active executive system could push adolescents toward greater risk taking. Such an interpretation is consistent with findings from Bjork et al. (2004) which showed adolescents have diminished striatal activation when they are anticipating responding for gains, but not upon receipt of reward. Thus, adolescents may experience more risky uncertain intermittent reinforcers as more rewarding compared to adults. The more salient the reward, the more likely a prefrontal dopaminergic response will occur that is sufficient to facilitate the formation of a conditioned association. Hence immaturity in executive-control neuro-maturational systems may put an adolescent at increased risk for substance use disorders and other types of risk-taking behaviors such as suicide attempts (Shaffer and Hicks, 1993; Costello et al., 2003). The data reported here are more consistent with the theory of Geier and Luna (2009), which states that adolescent risk taking may be best understood as an imbalance between inhibitory control, working memory, and reward systems that is biased toward short term goals (Geier and Luna, 2009). However, while our data do suggest increased activity of the frontal pole with age during decision making, these data do not suggest any association with age and Vstr during reward evaluation.

Our data have several limitations. We studied only very healthy high functioning adolescents. Therefore, our results may not be generalizable to population-based samples. Due to our sample size, we were unable to examine for gender differences. Additionally we did not study adults using the same task parameters so we were unable to directly compare healthy adolescent responses to behavioral risk with those of adults. However, although, we did not do physical examinations for pubertal stage, we were able to associate a proxy measure of maturity (i.e., age) with a decision-making brain ROI.

While our results came from a healthy high functioning nonmaltreated sample of adolescents, they point to the power of using a simple task (i.e., Decision-Reward Uncertainty task) for addressing types of risks and reward processing in children and adolescents with predisposing vulnerabilities. Given that the ability to evaluate risk and reward is a maturational process, it is important to examine the effects of early life stressors on these abilities. Conditions associated with maladaptive decision making and reward evaluation (e.g., substance use disorder) come to the fore during adolescence. A better understanding of the 
developmental progression of decision and reward networks will lead to more refined targets both for future research and for interventions.

\section{CONCLUSION}

We investigated functional brain activity in high functioning healthy adolescents while they performed the experimental Decision-Reward Uncertainty task (Huettel, 2006). Our analyses focused on the decision making or choice selection and outcome evaluation phases of decisions that involved behavioral risk (i.e., decision making under uncertainty). Behavioral risk trials evoked greater activation than the reward risk and no risk conditions in the anterior cingulate, medial frontal gyrus, dorsal lateral prefrontal cortex, bilateral frontal poles and inferior parietal lobes, precuneus, bilateral superior-middle frontal gyrus, IFG, and anterior insula. Our results were similar to those of young adults using the same task during behavioral risk (Huettel, 2006). During the behavioral and reward risk conditions regardless of reward outcome, overall mean frontal pole activity showed a positive correlation with age during the

\section{REFERENCES}

Anda, R. F., Croft, J. B., Felitti, V. J., Nordenberg, D., Giles, W. H., Williamson, D. F., and Giovino, G. A. (1999). Adverse childhood experiences and smoking during adolescence and adulthood. J. Am. Med. Assoc. 282, 1652-1658.

Angold, A., Prendergast, M., Cox, A., Harrington, R., Simonoff, E., and Rutter, M. (1995). The Child and Adolescent Psychiatric Assessment (CAPA). Psychol. Med. 25, 739-753.

Apicella, P., Ljungberg, T., Scarnati, E., and Schultz, W. (1991). Responses to reward in monkey dorsal and ventral striatum. Exp. Brain Res. 85, 491-500.

Bjork, J. M., Knutson, B., Fong, G. W., Caggiano, D. M., Bennett, S. M., and Hommer, D. W. (2004). Incentive-elicited brain activation in adolescents: similarities and differences from young adults. J. Neurosci. 24, 1793-1802.

Bjork, J. M., Smith, A. R., Danube, C. L., and Hommer, D. W. (2007). Developmental differences in posterior mesofrontal cortex recruitment by risky rewards. J. Neurosci. 27, 4839-4849.

Bolla, K. I., Eldreth, D. A., Matochik, J. A., and Cadet, J. L. (2005). Neural substrates of faulty decision-making in abstinent marijuana users. $\mathrm{Neu}$ roimage 26, 480-492.

Brainard, D. H. (1997). The psychophysics toolbox. Spatial Vision 10, 443-446.

Chambers, R. A., Taylor, J. R., and Potenza, M. N. (2003). Developmental neurocircuitry of motivation in adolescence: a critical period of addiction vulnerability. Am. J. Psy-

Costello, E. J., Mustillo, S., Erkanli, A., Keeler, G., and Angold, A. (2003). Prevalence and development of psychiatric disorders in childhood and adolescence. Arch. Gen. Psychiatry 60, 837-844.

Dahl, R. E. (2004). Adolescent brain development: a period of vulnerabilities and opportunities. Ann. N. Y. Acad. Sci. 1021, 1-22.

De Bellis, M. D. (2001). Developmental traumatology: a contributory mechanism for alcohol and substance use disorders Psychoneuroendocrinology 27, 155-170.

Delgado, M. R., Locke, H. M., Stenger, V. A., and Fiez, J. A. (2003). Dorsal striatum responses to reward and punishment: effects of valence and magnitude manipulations. Cogn. Affect. Behav. Neurosci. 3, 27-38.

Dillon, D. G., Holmes, A. J., Birk, J. L., Brooks, N., Lyons-Ruth, K., and Pizzagalli, D. A. (2009). Childhood adversity is associated with left basal ganglia dysfunction during reward anticipation in adulthood. Biol. Psychiatry 66, 206-213.

Elliott, R., Newman, J. L., Longe, O. A., and Deakin, J. F. (2003). Differential response patterns in the striatum and orbitofrontal cortex to financial reward in humans: a parametric functional magnetic resonance imaging study. J. Neurosci. 23, 303-307.

Ernst, M., and Mueller, S. V. (2008). The adolescent brain: insights from functional neuroimaging research. Dev. Neurobiol. 68, 729-743. chiatry 160, 1041-1052.

behavioral and reward risk conditions suggesting a developmental immaturity of this ROI. Additionally, reward response to the Decision-Reward Uncertainty task in adolescents was similar to that seen in young adults (Huettel, 2006). While our results came from a healthy high functioning non-maltreated sample of adolescents, this method (i.e., Decision-Reward Uncertainty task) can be used to address types of risks and reward processing in children and adolescents with predisposing vulnerabilities and add to the paucity of imaging studies of risk and reward processing during adolescence.

\section{ACKNOWLEDGMENTS}

The authors thank Lihong Wang, MD PhD for her assistance with this work. This work was supported by grants from the National Institute of Drug Abuse (RO1DA020989 and K24DA028773) and the National Institute of Mental Health (MH063407, K24MH071434) to Michael D. De Bellis and from the National Institute of Mental Health to Scott A. Huettel (RO1MH070685). Scott A. Huettel was supported by an Incubator Award from the Duke Institute for Brain Sciences.

Ernst, M., and Paulus, M. P. (2005). Neurobiology of decision making: a selective review from a neurocognitive and clinical perspective. Biol. Psychiatry 58, 597-604.

Ernst, M., Pine, D. S., and Hardin, M. (2006). Triadic model of the neurobiology of motivated behavior in adolescence. Psychol. Med. 36, 299-312.

Eshel, N., Nelson, E. E., Blair, J. R., Daniel, S. P., and Monique, E. (2007). Neural substrates of choice selection in adults and adolescents: development of the ventrolateral prefrontal and anterior cingulate cortices. Neuropsychologia 45 , 1270-1279.

Galvan, A., Hare, T. A., Parra, C. E., Penn, J., Voss, H., Glover, G., and Casey, B. J. (2006). Earlier development of the accumbens relative to orbitofrontal cortex might underlie risk-taking behavior in adolescents. J. Neurosci. 26, 6885-6892.

Geier, C., and Luna, B. (2009). The maturation of incentive processing and cognitive control. Pharmacol. Biochem. Behav. 93, 212-221.

Gogtay, N., Giedd, J. N., Lusk, L., Hayashi, K. M., Greenstein, D., Vaituzis, A. C., Nugent, T. F. III, Herman, D. H., Clasen, L. S., Toga, A. W., Rapoport, J. L., and Thompson, P. M. (2004). Dynamic mapping of human cortical development during childhood through early adulthood. Proc. Natl. Acad. Sci. U.S.A. 101, 8174-8179.

Guyer, A. E., Kaufman, J., Hodgdon, H. B., Masten, C. L., Jazbec, S., Pine, D. S., and Ernst, M. (2006). Behavioral alterations in reward system function: the role of childhood maltreatment and psychopathology. J. Am. Acad. Child Adolesc. Psychiatry 45, 1059-1067.

Huettel, S. A. (2006). Behavioral, but not reward, risk modulates activation of prefrontal, parietal, and insular cortices. Cogn. Affect. Behav. Neurosci. 6, 141-151.

Huettel, S. A., Song, A. W., and Mccarthy, G. (2005). Decisions under uncertainty: probabilistic context influences activation of prefrontal and parietal cortices. $J$. Neurosci. 25, 3304-3311.

Kaufman, J., Birmaher, B., Brent, D., Rao, U., Flynn, C., Moreci, P., Williamson, D., and Ryan, N. (1997). Schedule for affective disorders and schizophrenia for school-age children-present and lifetime version (K-SADS-PL): initial reliability and validity data. J. Am. Acad. Child Adolesc. Psychiatry 36, 980-988.

Kelley, A. E., Schochet, T., and Landry, C. F. (2004). Risk taking and novelty seeking in adolescence: introduction to part I. Ann. N. Y. Acad. Sci. 1021, 27-32.

Kerns, J. G., Cohen, J. D., Macdonald, A. W., Cho, R. Y., Stenger, V. A., and Carter, C. S. (2004). Anterior cingulate conflict monitoring and adjustments in control. Science 303, 1023-1026.

Kilpatrick, D. G., Acierno, R., Schnurr, P. P., Saunder, B., Resnick, H. S., and Best, C. L. (2000). Risk factors for adolescent substance abuse and dependence: data from a National Sample. J. Consult. Clin. Psychol. 68, 19-30. 
Konrad, K., Neufang, S., Thiel, C. M., Specht, K., Hanisch, C., Fan, J., Herpertz-Dahlmann, B., and Fink, G. R. (2005). Development of attentional networks: an fMRI study with children and adults. Neuroimage 28, 429-439.

Kwon, H., Reiss, A. L., and Menon, V. (2002). Neural basis of protracted developmental changes in visuospatial working memory. Proc. Natl. Acad. Sci. U.S.A. 99, 13336-13341.

Lee, H.-L., Devlin, J. T., Shakeshaft, C., Stewart, L. H., Brennan, A., Glensman, J., Pitcher, K., Crinion, J., Mechelli, A., Frackowiak, R. S. J., Green, D. W., and Price, C. J. (2007). Anatomical traces of vocabulary acquisition in the adolescent brain. J. Neurosci. 27, 1184-1189.

Martin-Santos, R., Fagundo, A. B., Crippa, J. A., Atakan, Z., Bhattacharyya, S., Allen, P., Fusar-Poli, P., Borgwardt, S., Seal, M., Busatto, G. F., and Mcguire, P. (2010). Neuroimaging in cannabis use: a systematic review of the literature. Psychol. Med. 40, 383-398.

May, J. C., Delgado, M. R., Dahl, R. E., Stenger, V. A., Ryan, N. D., Fiez, J. A., and Carter, C. S. (2004). Eventrelated functional magnetic resonance imaging of reward related brain circuitry in children and adolescents. Biol. Psychiatry 55, 359-366.

Mehta, M., Gore-Langton, E., Golembo, N., Colvert, E., Williams, S., and Sonuga-Barke, E. (2010). Hyporesponsive reward anticipation in the basal ganglia following severe institutional deprivation early in life. J. Cogn. Neurosci. 10, 2316-2325.

Morgan, D., Grant, K. A., Gage, H. D., Mach, R. H., Kaplan, J. R., Prioleau, O., Nader, S. H., Buchheimer, N., Ehrenkaufer, R. L., and Nader, M. A. (2002). Social dominance in monkeys: dopamine D2 receptors and cocaine self-administration. Nat. Neurosci. 5, 169-174.

O’Doherty, J., Kringelbach, M. L., Rolls, E. T., Hornak, J., and Andrews, C. (2001). Abstract reward and punishment representations in the human orbitofrontal cortex. Nat. Neurosci. 4, 95-102.

Papp, M., Klimek, V., and Willner, P. (1994). Parallel changes in dopamine D2 receptor binding in limbic forebrain associated with chronic mild stressinduced anhedonia and its reversal by imipramine. Psychopharmacology (Berl.) 115, 441-446.

Paulus, M. P., Hozack, N., Zauscher, B., Mcdowell, J. E., Frank, L., Brown, G. G., and Braff, D. L. (2001). Prefrontal, parietal, and temporal cortex networks underlie decision-making in the presence of uncertainty. $\mathrm{Neu}$ roimage 13, 91-100.

Rangel, A., Camerer, C., and Montague, P. R. (2008). A framework for studying the neurobiology of value-based decision making. Nat. Rev. Neurosci. 9, 545-556.

Roesch, M. R., and Olson, C. R. (2004). Neuronal activity related to reward value and motivation in primate frontal cortex. Science 304 307-310.

Schott, B. H., Minuzzi, L., Krebs, R. M., Elmenhorst, D., Lang, M., Winz, O. H., Seidenbecher, C. I., Coenen, H. H., Heinze, H.-J., Zilles, K., Düzel, E., and Bauer, A (2008). Mesolimbic functional magnetic resonance imaging activations during reward anticipation correlate with reward-related ventral striatal dopamine release. J. Neurosci. 28, 14311-14319.

Schultz, W., Tremblay, L., and Hollerman, J. R. (2000). Reward processing in primate orbitofrontal cortex and basal ganglia. Cereb. Cortex 10, 272-284.

Shaffer, D., and Hicks, R. (eds). (1993). The Epidemiology of Child and Adolescent Suicide. New York, NY: Oxford University Press, Inc.

Siegel, J., and Shaughnessy, M. F. (1995). There's a first time for everything: understanding adolescence. Adolescence 30, 217-221.

Spear, L. P. (2000). The adolescent brain and age-related behavioral manifestations. Neurosci. Biobehav. Rev. 24 417-463.

Verdejo-Garcia, A., Benbrook, A., Funderburk, F., David, P., Cadet, J. L. and Bolla, K. I. (2007). The differential relationship between cocaine use and marijuana use on decisionmaking performance over repeat testing with the Iowa gambling task. Drug Alcohol Depend. 90, 2-11.
Wechsler, D. (1991). Wechsler Intelligence Scale for Children, 3rd Edn. San Antonio: The Psychological Corporation.

Xiangrui, L., Zhong-Lin, L. D'argembeau, A., Ng, M., and Bechara, A. (2010). The Iowa gambling task in fMRI Images. Hum. Brain Mapp. 31, 410-423.

Conflict of Interest Statement: The authors declare that the research was conducted in the absence of any commercial or financial relationships that could be construed as a potential conflict of interest.

Received: 24 December 2010; accepted: 16 November 2011; published online: 19 December 2011.

Citation: Yaxley RH, Van Voorhees EE, Bergman S, Hooper SR, Huettel SA and De Bellis MD (2011) Behavioral risk elicits selective activation of the executive system in adolescents: clinical implications. Front. Psychiatry 2:68. doi 10.3389/fpsyt.2011.00068

This article was submitted to Frontiers in Child and Neurodevelopmental Psychiatry, a specialty of Frontiers in Psychiatry. Copyright (C) 2011 Yaxley, Van Voorhees, Bergman, Hooper, Huettel and De Bellis. This is an open-access article distributed under the terms of the Creative Commons Attribution Non Commercial License, which permits non-commercial use, distribution, and reproduction in other forums, provided the original authors and source are credited. 